\title{
LOW TEMPERATURE QUALITY FACTOR SCALING OF GHz FREQUENCY SILICON RESONATORS \\ Eugene Hwang ${ }^{*}$ and Sunil A. Bhave \\ OxideMEMS Lab, Cornell University, Ithaca, NY, USA
}

\begin{abstract}
In this paper, we demonstrate quality factor $(Q)$ enhancement of a silicon RF MEMS resonator by cryogenic cooling from room temperature down to liquid nitrogen temperatures (78K). The resulting $Q$ is approximately 63,000 at a frequency of $3.72 \mathrm{GHz}$, yielding an $f-Q$ product of $2.34 \times 10^{14} \mathrm{~Hz}$. This work verifies that the resonator is operating in the Landau-Rumer regime at room temperature, where individual phonon scattering results in a strong dependence of $Q$ on temperature [1-3]. We find that as the temperature approaches $120 \mathrm{~K}$, the $Q$ converges to a constant value, indicating that intrinsic phonon loss mechanisms are replaced by temperature independent electrical and mechanical anchor losses as the dominant $Q$ limiting factor.
\end{abstract}

\section{INTRODUCTION}

Advances in micromechanical resonators have resulted in high quality factor $(Q)$ resonators at room temperature with frequencies in the microwave regime, highlighting the possibility of their use in integrated RF systems [4, 5]. Theoretical and experimental studies hypothesize that phonon interactions dominate the intrinsic loss in bulk-mode resonators, making possible even higher $Q$ at lower temperatures [1-3, 6-12]. Chip-scale micro-cryogenic cooling is not as farfetched as it seems, as integrated microsystems demonstrating cooling to temperatures as low as $76 \mathrm{~K}$ have already been developed [13]. Recent measurements of $Q$ in $60-\mathrm{MHz}$ silicon resonators demonstrated $Q \propto 1 / \mathrm{T}$, characteristic of the Akhiezer effect [7-9]. Instead, if we operate at frequencies in the Landau-Rumer regime, $Q$ $\propto 1 / \mathrm{T}^{4}$, greatly enhancing the gains in $Q$ due to cooling. We have previously demonstrated a pn-diode transduced, low motional impedance resonator (Fig. 1) at $3.72 \mathrm{GHz}$, which is operated in the Landau-Rumer regime [14]. This transduction mechanism is used primarily because it allows for efficient transduction at high frequencies in a completely homogeneous resonator, allowing us to carefully study the intrinsic loss mechanisms due to phonon scattering.

\section{EXPERIMENTAL RESULTS AND DISCUSSION}

An acoustic wave in a resonator can be thought of as a highly excited acoustic phonon mode containing a number of phonons much larger than the thermal equilibrium value. Due to various scattering processes, phonons in this highly excited mode will decay

Table 1: Expressions estimating $Q$ limits due to the two main phonon-phonon scattering mechanisms [1-3, 7-9]

\begin{tabular}{|c|c|}
\hline $\begin{array}{c}\text { Akhiezer } \\
\text { Effect }\end{array}$ & $Q_{A K E}=\frac{2 \pi \rho c_{D}{ }^{2}}{c_{v} \gamma^{2}} \frac{1+\left(\omega \tau_{t h}\right)^{2}}{\tau_{t h}} \frac{1}{\omega T}$ \\
& $\tau_{t h}=\frac{3 \kappa}{c_{v} c_{D}^{2}}$ \\
\hline $\begin{array}{c}\text { Landau- } \\
\text { Rumer } \\
\text { Effect }\end{array}$ & $Q_{L-R}=\frac{30 \rho c_{l}^{5} h^{3}}{\pi^{4} \gamma^{2} k_{B}^{4}} \frac{1}{T^{4}}$ \\
\hline$\rho=$ density & $\gamma=$ Grüneisen parameter \\
$c_{l}=$ longitudinal velocity & $\kappa=$ thermal conductivity \\
$c_{D}=$ Debye velocity & $h=$ Planck's constant \\
$c_{v}=$ volumetric heat capacity & \\
\hline
\end{tabular}

(a)

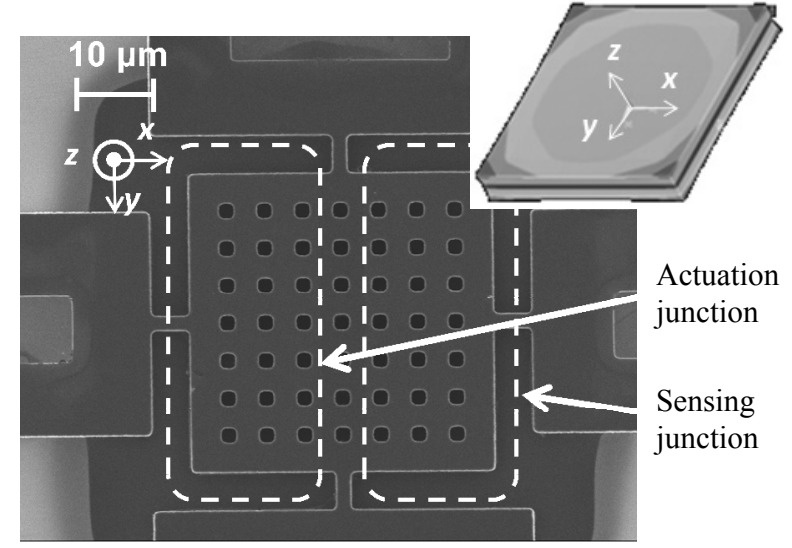

(b)

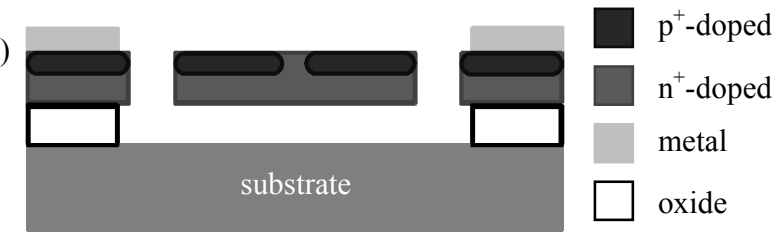

Figure 1. (a) Top-view SEM of the pn-diode transduced 3.7-GHz silicon micromechanical resonator and ANSYS simulation of thickness-stretch (FBAR) mode (inset) [14] (b) resonator cross sectional diagram

into other modes, resulting in attenuation of the acoustic wave. The dominant phonon-phonon scattering mechanisms depend strongly on the relationship between $\omega$, the frequency of the acoustic wave, and the thermal phonon lifetime $\tau_{t h}$. When $\omega \tau_{t h}<<1$, the device is in the Akhiezer regime and when $\omega \tau_{t h}>1$, the Landau-Rumer effect

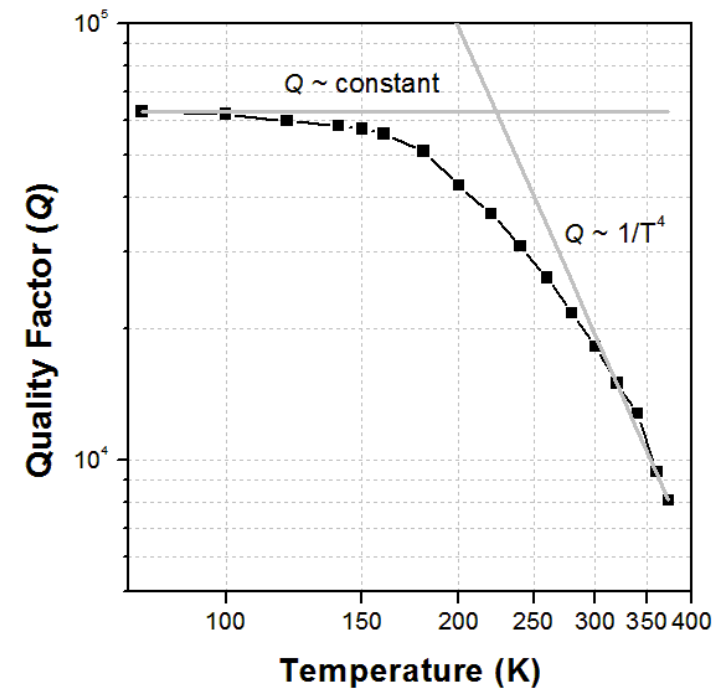

Figure 2. Measured $Q$ of pn-diode transduced silicon resonator showing $1 / T^{4}$ dependence at temperatures close to $300 \mathrm{~K}$. The $Q$ eventually tapers off to a constant value at around $120 \mathrm{~K}$ to a maximum of about 63,000. We believe this is due to anchor losses and routing resistance. All measurements are performed in a Lakeshore vacuum $R F$ probe station. 


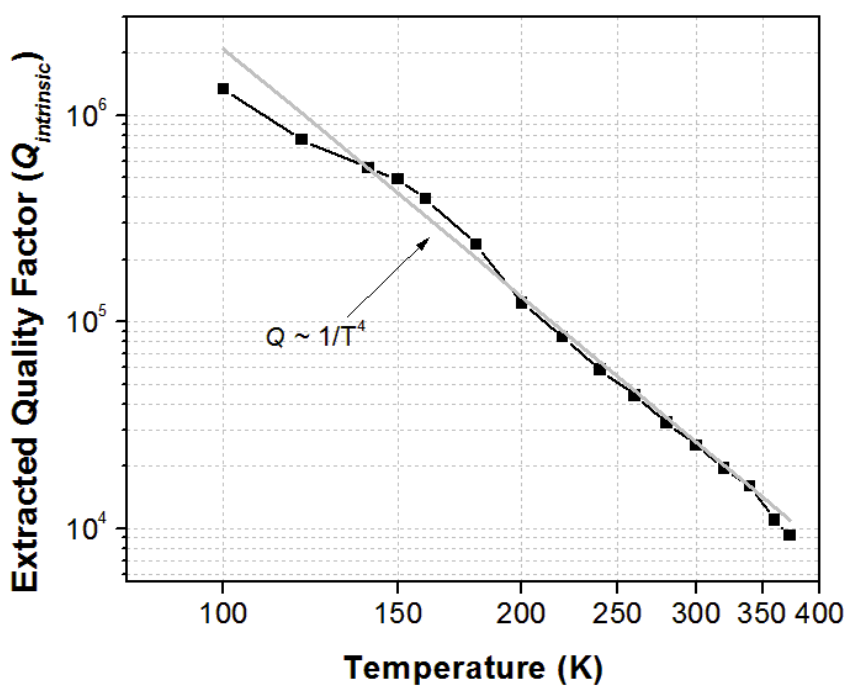

Figure 3. Extracted $Q$ of pn-diode transduced silicon resonator by removing lumped anchor losses using relationship given by Eq. (3). A near $1 / T^{4}$ dependence is seen throughout the measured temperature range indicating operation in the Landau-Rumer regime.

must be considered. Acoustic attenuation in both regimes has been studied extensively and theoretical details can be found in the literature [1-3, 7-9]. Expressions for the quality factor in these regimes are shown in Table 1.

Interestingly, it has been shown that for resonators operating in the Landau-Rumer regime, $Q$ is constant with frequency, a fact which is exploited in the high frequency resonators used in this work [14]. Operation in this regime is beneficial not only in terms of $f-Q$ product scaling with frequency $(f-Q \propto f)$, but also with temperature due to the strong dependence of $Q \propto 1 / T^{4}$, as shown in Eq. (2). Fig. 2 verifies that our device is in this regime by showing such dependence at temperatures close to $300 \mathrm{~K}$. However, the $Q$ approaches a constant value as temperature decreases, which we believe to be a result of both electrical and mechanical losses due to the anchor. Electrical resistive losses, which typically are not considered in electrostatically transduced resonators, are important because of the low motional impedance of these devices.

In order to verify whether the remaining loss is due to the Landau-Rumer effect, we lumped the $Q$ obtained in the constant- $Q$ region into an equivalent anchor loss term $Q_{\text {anchor }}$ and, assuming that the remaining loss is entirely intrinsic, used the relationship

$$
\frac{1}{Q_{\text {total }}}=\frac{1}{Q_{\text {anchor }}}+\frac{1}{Q_{\text {intrinsic }}}
$$

to determine $Q_{\text {intrinsic }}$ plotted in Fig. 3. This plot shows a $1 / \mathrm{T}^{4}$ dependence, thus verifying operation in the Landau-Rumer regime. Note that these experiments were repeated with three other similar resonators, all showing similar temperature dependence.

\section{CONCLUSION}

Through this work, we were able to verify operation of the pn-diode transduced micromechanical resonators in the Landau-Rumer regime. We have demonstrated the $1 / \mathrm{T}^{4}$ dependence of $Q$ and exploited it to demonstrate $Q \sim 63,000$ and $f-Q$ product of $2.34 \times 10^{14} \mathrm{~Hz}$ at $78 \mathrm{~K}$. The conclusions drawn from this work are not dependent on the use of pn-diode transduction and are widely applicable to $\mathrm{GHz}$ frequency single crystal silicon resonators. With the availability of chip-scale spot cooling, silicon micromechanical resonators show the potential to be used in high- $Q$ applications such as chip-scale spectrum analysis and narrowband RF applications.

\section{ACKNOWLEDGEMENTS}

This work was funded by the DARPA HI-MEMS project and Army Research Labs (ARL). The devices were fabricated in the Cornell Nanoscale Science and Technology Facility (CNF).

\section{REFERENCES}

[1] H. J. Maris, "Interaction of sound waves with thermal phonons in dielectric crystals," In W. P. Mason, R. N. Thurston, editors, Physical Acoustics - Volume VIII, Academic Press, New York, 1971.

[2] R. Tabrizian, M. Rais-Zadeh, and F. Ayazi, "Effect of phonon interactions on limiting the $f . Q$ product of micromechanical resonators," Transducers '09, Denver, CO, USA, June 21-25, 2009, pp. 2131-2134.

[3] P. G. Klemens, "Decay of high-frequency longitudinal phonons," J. Appl. Phys., 38, 12 (1967).

[4] D. Weinstein and S. A. Bhave, "The resonant body transistor," accepted for publication in Nano Letters, 2010.

[5] M. Rinaldi, C. Zuniga, and G. Piazza, "5-10 GHz AlN contour-mode nanoelectromechanical resonators," MEMS 2009, Sorrento, Italy, Jan. 25-29, 2009, pp. 916-919.

[6] S. A. Chandorkar et al, "Limits of quality factor in bulk-mode micromechanical resonators," MEMS 2008, Tucson, AZ, USA, Jan. 13-17, 2008, pp. 74-77.

[7] T. O. Woodruff and H. Ehrenreich, "Absorption of sound in insulators," Physical Review B, 123, 5 (1961).

[8] W. P. Mason and T. B. Bateman, "Ultrasonic-wave propagation in pure silicon and germanium," J. Acoust. Soc. Am., 36, 4 (1964).

[9] A. N. Cleland, Foundations of Nanomechanics: From Solid-State Theory to Device Applications, Springer-Verlag, Berlin, 2003.

[10] A. El Habti, F. Bastien, "Low temperature limitation on the quality factor of quartz resonators," IFCS 1992, May 27-29, 1992, pp. 597-602.

[11] B. Kim et al, "Temperature dependence of quality factor in MEMS resonators," MEMS 2006, Istanbul, Turkey, Jan. 22-26, 2006, pp. 590-593.

[12] W.-C. Li, Y. Lin, B. Kim, Z. Ren, and C. T.-C. Nguyen, "Quality factor enhancement in micromechanical resonators at cryogenic temperatures," Transducers '09, Denver, CO, USA, June 21-25, 2009, pp. 1445-1448.

[13] M.-H. Lin, P. E. Bradley, H.-J. Wu, J. C. Booth, R. Radebaugh, Y. C. Lee, "Design, fabrication, and assembly of a hollow-core fiber-based micro cryogenic cooler," MEMS 2009, Sorrento, Italy, Jan, 25-29, 2009, pp. 1114-1117.

[14] E. Hwang and S. A. Bhave, "PN-diode transduced 3.7-GHz silicon resonator," MEMS 2010, Hong Kong SAR, Jan. 24-28, 2010, pp. 208-211.

\section{CONTACT:}

*E. Hwang, tel: +1-408-515-4132; eoh3@cornell.edu 